\title{
Abordaje diagnóstico de la enfermedad glomerular del adulto Transformando una idea compleja en un proceso práctico
}

\author{
Diagnostic approach of glomerular disease in \\ adults \\ Transforming a complex idea in a practical process
}

Leidy González, Jorge Cantillo • Bogotá, D.C. (Colombia)

\begin{abstract}
"Esta publicación es el fruto del agradecimiento a mis exprofesores (1994-1996) Hernando Altahona S, Carlos Rosselli S, Mauricio Ruiz M. y Francisco J. Barreto S. (Q.E.P.D.) quien partió al mundo espiritual el 26 de Enero de 2013 y que me enseñaron la importancia del análisis clínico nefrológico en beneficio de nuestros pacientes".

Jorge CANTILLO TuRBAY
\end{abstract}

\section{Introducción}

El término glomerulonefritis o glomerulopatías se utiliza en forma amplia para designar las enfermedades que afectan la estructura y función glomerular, tratándose de entidades clínicas heterogéneas tanto en su etiología, manifestaciones, curso clínico y pronóstico (1), pero que tienen como denominador común el hallazgo histológico de inflamación del penacho glomerular (salvo cuatro excepciones puntuales que son la nefropatía diabética, nefroesclerosis hipertensiva, amiloidosis y nefropatías hereditarias) y que tienen implicado un mecanismo inmune en su fisiopatología (2). El rápido y eficaz reconocimiento de la causa de la enfermedad glomerular resultará en una aproximación terapéutica racional, segura y efectiva con especial impacto en preservación de función renal (3), teniendo que mencionar que a nivel mundial, la enfermedad glomerular primaria es la segunda causa de enfermedad renal crónica terminal, precedida solamente por la nefropatía diabética la cual es finalmente una glomerulopatía secundaria (1).

\section{¿Cómo aproximarse al diagnóstico de enfermedad glomerular?}

Tradicionalmente se han empleado diversos sistemas de clasificación para aproximarse al estudio de la enfermedad glomerular, a continuación se describe un proceso de cuatro pasos, que en concepto de los autores resulta ser metódico y permite simplificar el proceso diagnóstico:

- Establecer que síndrome de presentación de enfermedad glomerular tiene.

- Recolectar datos clínicos relevantes para establecer una probable correlación clínico patológica.

- Determinar la utilidad de los datos de laboratorio e imágenes.

- Establecer el diagnóstico etiológico. ¿Existe indicación de biopsia renal?

\section{Síndrome nefrótico}

\section{1. ¿Qué síndrome de presentación tiene?}

Se define en presencia de proteinuria en rango nefrótico ( $>3.5 \mathrm{~g} / 24 \mathrm{horas} / 1.73 \mathrm{~m} 2 \mathrm{SCT})$, en asociación con edema, hipoalbuminemia $\leq 3 \mathrm{~g} / \mathrm{dL}$ e hipercolesterolemia; característicamente no hay HTA, ni deterioro en tasa de filtración glomerular tan marcados como en el síndrome nefrítico (4). Aunque escapa al propósito de esta revisión hacer énfasis en la
Dra. Leidy González Rojas: Residente Medicina Interna III año, Universidad Nacional de Colombia; Dr. Jorge Cantillo Turbay: Internista Nefrólogo. Profesor Asociado Departamento de Medicina Interna,Facultad de Medicina, Universidad Nacional de Colombia. Docente Clínico, Facultades de Medicina, Universidad de La Sabana y Universidad El Bosque. Unidad Renal Hospital Occidente de Kennedy y Centro Policlínico del Olaya S.A. Bogotá D.C., Colombia.

Correspondencia. Dr. Jorge Cantillo Turbay E-mail: md_cantillo@yahoo.com

Recibido: 28/XII/2012 Aceptado: 7/III/2013 
fisiopatología, resulta útil entender el síndrome nefrótico como una podocitopatía, entendiéndose por este término el daño, la disfunción o la pérdida de podocitos que resulta en proteinuria masiva (5). Ahora, de forma global los tres patrones histológicos que más frecuentemente derivan del daño del podocito en el adulto son: glomeruloesclerosis focal y segmentaria (35\%), nefropatía membranosa (33\%) y enfermedad de cambios mínimos (15\%), con algunas diferencias en incidencia de acuerdo con la raza y a áreas geográficas $(4,5)$. Cada una de ellas tiene diferencias en su evolución y podrá ser de etiología primaria o secundaria, para lo cual resultarán útiles los datos de correlación clínica y de laboratorio que se presentarán más adelante. Dentro de las causas secundarias tiene papel protagónico la nefropatía diabética que sigue siendo de forma global la causa más frecuente de síndrome nefrótico del adulto pudiendo presentarse con cualquiera de los tres tipos histológicos de enfermedad glomerular que afectan el podocito. Otras etiologías secundarias importantes son la nefropatía lúpica tipo $\mathrm{V}$ con patrón histológico de nefropatía membranosa (6); amiloidosis, infecciones por malaria, virus hepatotrópos B y C, VIH (diversos patrones histológicos), múltiples medicamentos, mieloma múltiple, etiologías para las cuales deberá hacerse el enfoque respectivo y las nefropatías paraneoplásicas a las que nos referiremos más adelante.

\section{Síndrome nefrítico}

Se caracteriza por el inicio súbito de hematuria micro o macroscópica, oliguria, falla renal aguda, manifestándose con retención hídrica, edema e hipertensión arterial (7). La hematuria suele acompañarse de presencia en orina de cilindros eritrocitarios y sedimento telescopado que son la marca de la inflamación glomerular (8). Aunque puede haber proteinuria, no suele ser tan marcada como en el síndrome nefrótico, siendo habitualmente inferior a $2 \mathrm{~g} / 24$ horas/1.73m2 SCT (8). En contraste con el síndrome nefrótico donde prevalece la lesión del podocito, en el síndrome nefrítico predominan las lesiones inflamatorias del glomérulo comprometiendo células mesangiales, endoteliales y/o células parietales epiteliales, en relación más frecuentemente con patrones histológicos de glomerulonefritis membranoproliferativa, nefropatía por IgA, glomerulonefritis proliferativa endocapilar, glomerulonefritis proliferativa extracapilar o crescéntica (9), habitualmente en relación con etiologías secundarias. Son múltiples las etiologías relacionadas pero en términos prácticos podrían clasificarse en tres grupos: relacionadas con anticuerpos, relacionadas con complejos inmunes o las de mecanismo pauciinmune, siendo la intensidad y el tiempo de la respuesta inflamatoria los que definen el curso de la enfermedad como síndrome nefrítico o como glomerulonefritis rápidamente progresiva (9).

\section{Glomerulonefritis rápidamente progresiva}

Forma menos frecuente (2\%) pero más agresiva de glomerulonefritis activa, constituyendo una verdadera emergencia médica que se manifiesta como síndrome nefróticonefrítico mixto asociado a rápida declinación de la función renal (aumento de creatinina $>2 \mathrm{mg} / \mathrm{dL}$ en menos de tres meses o duplicación de la creatinina sérica en tres meses o debut de enfermedad glomerular con creatinina $>3 \mathrm{mg} / \mathrm{dL}$ ), tamaño renal conservado o aumentado, alta frecuencia de uremia al inicio del cuadro clínico y poca tendencia a la recuperación espontánea (10). Las mismas causas que llevan a síndrome nefrítico son responsables de inducir glomerulonefritis rápidamente progresiva con un patrón histológico de glomerulonefritis proliferativa extracapilar o crescéntica $(9,10)$. Las causas se pueden dividir en relacionadas con anticuerpos (20\%), relacionadas con complejos inmunes (30\%) o las de mecanismo pauciinmune $(50 \%)(10,11)$ (Tabla 1$)$.

Tabla 1. Ejemplos de glomerulopatías rápidamente progresivas.

\begin{tabular}{|c|c|c|}
\hline Mecanismo & Descripción & Enfermedad \\
\hline \multirow{2}{*}{$\begin{array}{l}\text { Anticuerpos anti- membrana basal } \\
\text { glomerular (anti-MBG) }\end{array}$} & Con compromiso pulmonar & Síndrome Goodpasture \\
\hline & Sin compromiso pulmonar & Enfermedad anti-MBG \\
\hline \multirow[t]{4}{*}{ Complejos inmunes } & Anticuerpos antinucleares & LES lupus eritematoso sistémico \\
\hline & Anticuerpos dirigidos contra un patógeno específico & Glomerulonefritis posinfecciosa \\
\hline & Anticuerpos contra antígenos celulares & $\begin{array}{l}\text { Púrpura Henoch-Schölein, } \\
\text { Nefropatía IgA }\end{array}$ \\
\hline & Crioglobulinas & Glomerulonefritis membranoproliferativa asociada a VIH, a VHB, a VHC \\
\hline Pauciinmune & ANCA & $\begin{array}{l}\text { Granulomatosis de Wegener } \\
\text { Poliarteritis microscópica } \\
\text { Glomerulonefritis rápidamente progresiva idiopática } \\
\text { Síndrome de Churg Strauss }\end{array}$ \\
\hline
\end{tabular}




\section{Hematuria asintomática}

Se refiere a la presencia de hematuria micro o macroscópica en pacientes con tasa de filtración glomerular normal sin evidencia de enfermedad sistémica ni compromiso renal, aunque algunos pueden tener proteinuria inferior a $1.5 \mathrm{~g} / 24$ horas. Tiene características de hematuria de origen alto en el sedimento urinario con eritrocitos dismórficos, acantocitos y cilindros eritrocitarios. El diagnóstico diferencial incluye más comúnmente causas urológicas; de todos los casos de hematuria sin proteinuria sólo el $19 \%$ tienen origen glomerular en asociación en orden de frecuencia a patrones histológicos de nefropatía por IgA, enfermedad de membrana basal delgada, glomerulonefritis proliferativa focal y enfermedades congénitas como el síndrome de Alport $(7,12)$.

\section{Proteinuria asintomática}

Proteinuria inferior a $1 \mathrm{~g} / 24$ horas sin hallazgos de enfermedad sistémica ni compromiso de la función renal. Puede ser transitoria funcional por alteración de la membrana basal glomerular en estados de bajo volumen circulante eficaz, fiebre, ejercicio extenuante; transitoria ortostática en adolescentes; persistente por sobreflujo- sobreproducción como en rabdomiolisis o en mieloma múltiple (sin ser este el único mecanismo de compromiso glomerular por mieloma múltiple); persistente de origen tubulointersticial como en los casos de nefroesclerosis hipertensiva y nefritis tubulointersticial aguda/ crónica; persistente glomerular en estadíos tempranos de la enfermedad de cambios mínimos $(1,7)$.

\section{2. ¿Cuáles son los datos clínicos relevantes para establecer una probable correlación clinicopatológica?}

Conocer las características clínicas asociadas a los diferentes patrones histológicos. A continuación se describen las características clínicas habituales asociadas a los diferentes patrones histológicos de enfermedad glomerular de etiología primaria o idiopática, de manera que los casos que difieran francamente de ese comportamiento deben alertar la posibilidad de tener implicada una etiología secundaria. (Tabla 2)

\section{Glomeruloesclerosis focal y segmentaria}

De etiología primaria en $60 \%$ de los casos, secundaria en $40 \%$. La forma primaria o idiopática suele iniciar entre la tercera y cuarta década de la vida predomina en género masculino, 70\% debutan con síndrome nefrótico con proteinuria habitualmente $\leq 10 \mathrm{~g} / 24$ horas (proteinuria en mayor rango suele estar asociada a etiologías secundarias); en 50\% de los casos asociado a HTA y hematuria microscópica y, en 30\% con algún grado de insuficiencia renal (a diferencia de la nefropatía membranosa en la cual no es común encontrar insuficiencia renal ni HTA) $(13,14)$. La forma secundaria ocurre a cualquier edad en relación con patologías que aumentan la presión intraglomerular (enfermedad renovascular, obesidad) o en condiciones que se acompañen de disminución en la carga de nefronas como en estados posnefrectomía. La historia natural de la enfermedad primaria muestra remisión espontánea en menos de 5\% de los casos, siendo lo habitual sin tratamiento que 50\% lleguen a enfermedad renal crónica terminal en los siguientes seis años, con mención especial al subtipo histológico colapsante que predomina en afroamericanos y en infección por $\mathrm{VIH}$, donde la progresión a ERC-T es mucho más rápida en el lapso de pocos meses (14).

\section{Glomerulopatía membranosa}

Es la principal causa de síndrome nefrótico en raza blanca. Ochenta por ciento de los casos son de etiología primaria o idiopática versus $20 \%$ de etiología secundaria. Tiene predilección por el género masculino con relación 3:1 y presentación de distribución bifásica siendo la forma primaria más comúnmente encontrada entre los 30 y 40 años de edad y un segundo pico de presentación que ocurre entre los 50-60 años de edad en asociación importante con glomerulopatía paraneoplásica (15). Otras causas importantes de nefropatía membranosa secundaria son lupus eritematoso (nefritis lúpica tipo $\mathrm{V}$ ), infecciones, medicamentos (se ampliará al respecto más adelante en el texto). Las formas primarias se manifiestan en el $90 \%$ de los casos con proteinuria masiva, habitualmente $>10-12 \mathrm{~g} / 24$ horas, $30 \%$ con hematuria microscópica y no es

Tabla 2. Características de las glomerulopatías agrupadas por patrones histológicos.

\begin{tabular}{|c|c|c|c|c|c|c|}
\hline Patrón histológico & S. nefrótico & S. nefrítico & Hematuria & daño renal & HTA & Edad (años) \\
\hline ECM & ++++ & & & Rara & Rara & 20 \\
\hline NM & ++++ & & & Rara* & Rara & $30-40$ \\
\hline GEFS & +++ & & & Común & Común & $20-30$ \\
\hline GMP & ++ & +++ & + & Común & Común & $8-30$ \\
\hline N. IgA & & ++ & +++ & Común & Variable & $10-30$ \\
\hline GRP & +++ & +++ & & Siempre & Común & Variable \\
\hline GPE & + & ++++ & & Muy frecuente & Común & Variable \\
\hline
\end{tabular}


habitual encontrar HTA, ni compromiso en tasa de filtración glomerular, ni en niveles de creatinina sérica (a menos que esté manifestando complicaciones derivadas del síndrome nefrótico como trombosis de vena renal o falla renal aguda por excesiva diuresis o nefritis intersticial por diuréticos o por sepsis, que no son infrecuentes) (9).

\section{Enfermedad de cambios mínimos}

Es la tercera causa de síndrome nefrótico del adulto, sin predilección de género, siendo la edad de presentación habitual en el adulto joven (promedio 20 años). Más de $90 \%$ de los casos son de etiología primaria, con el restante $10 \%$ asociado a medicamentos, alergia (casos reportados con inmunizaciones) y malignidad hematológica. Se caracteriza por el inicio abrupto de proteinuria $3-20 \mathrm{~g} / \mathrm{dí} / 1.73 \mathrm{~m}^{2} \mathrm{con}$ síndrome nefrótico plenamente manifiesto, sin HTA o leve incremento, sin elevación en azoados, con sedimento urinario normal o hematuria microscópica, con rápida respuesta favorable al manejo conservador o con corticoides.

\section{Glomerulonefritis membranoproliferativa}

Suele manifestarse como síndrome nefrítico de inicio súbito o síndrome nefrítico-nefrótico mixto, con hipocomplementemia sostenida $>8$ semanas, anemia sin correlación con la tasa de filtración glomerular. Tiene pico de incidencia entre 8-30 años de edad, más comúnmente de etiología secundaria ( $>80 \%$ casos), siendo el prototipo de glomerulopatía por depósito de complejos inmunes. Dentro del $20 \%$ que son de etiología primaria, 5-15\% pueden explicar los síndromes nefróticos primarios del adulto. Se clasifica en tres subtipos histológicos de acuerdo con el sitio donde se depositan los complejos inmunes, determinando el espectro y la severidad de las manifestaciones clínicas. 50-80\% de los casos tienen HTA marcada, sedimento activo telescopado y $25 \%$ de los pacientes presentan curso de glomerulonefritis crónica progresiva. Dado que es el prototipo de enfermedad glomerular por complejos inmunes, ante la sospecha de glomerulonefritis membranoproliferativa se deberá individualizar la evaluación de cada paciente buscando probabilidad o no de cuatro grupos fisiopatológicos grandes: mediado por anticuerpos antinucleares como en LES, anticuerpos antipatógeno como en las glomerulonefritis posinfecciosas, antígenos celulares como en nefropatía por IgA secundaria y púrpura de Henoch Scholein, crioglobulinemia por infección por VHB, $\operatorname{VHC}(2,10,16)$.

\section{Nefropatía IgA}

Consiste en el daño inflamatorio por depósito mesangial de IgA, siendo $80 \%$ de los casos idiopáticos y $20 \%$ de etiología secundaria a múltiples enfermedades hepáticas, intestinales, dermatológicas, pulmonares e inmunológicas sistémicas. La forma primaria es más frecuente entre la segunda y tercera décadas de la vida, con predilección por el género masculino en relación 2-6:1. Su forma de presentación habitual, 40-50\% de los casos, consiste en hematuria macroscópica episódica usualmente 24 horas siguientes a una infección respiratoria alta (a diferencia de la hematuria de etiología posestreptocica que aparece 2-3 semanas después del episodio infeccioso); $30-40 \%$ de los casos tendrán hematuria microscópica asintomática. Sólo 5\% de los casos desarrollan síndrome nefrótico y $\leq 2 \%$ presentan HTA y daño renal crónico. En los casos de etiología primaria suele manifestarse entonces como hematuria episódica que autolimita, a diferencia de las de etiología secundaria en donde suele manifestarse con síndrome nefrítico con diferentes grados de severidad $(10,17)$.

\section{Glomerulonefritis proliferativa endocapilar}

El término engloba una serie de enfermedades glomerulares caracterizadas histológicamente por hipercelularidad del ovillo glomerular especialmente de las células mesangiales, sin cambios en la membrana basal, asociado a grados variables de infiltración por polimorfonucleares que da lugar a la obliteración de las luces capilares; en casos de mayor severidad existe proliferación de células epiteliales y formación de semilunas. Generalmente están asociadas a etiologías infecciosas que producen complejos antígenoanticuerpo nefritogénicos, siendo el prototipo de ellas la glomerulonefritis postestreptocócica. La sintomatología es variable, siendo lo habitual síndrome nefrítico, pero en algunos casos puede cursar sólo con hematuria microscópica, o por el contrario en el espectro de severidad, cursar con glomerulonefritis rápidamente progresiva. Las infecciones más frecuentemente implicadas incluyen infecciones orofaríngeas o en piel por estreptococos, endocarditis por Stafilococcus aureus, Streptococcus viridans, abscesos viscerales por S. Aureus, Enterobacter spp, Pseudomonas spp, Proteus mirabilis, neumonía por Mycoplasma, fiebre tifoidea (Salmonella typhi); infecciones virales por virus de Epstein Barr, Parvovirus B19, Cossackie, rubéola, hepatitis $\mathrm{B}$ y algunas infecciones parasitarias.

\section{Glomerulonefritis proliferativa extracapilar o crescéntica}

Corresponde al patrón histológico de las glomerulonefritis rápidamente progresivas, síndrome del cual ya se ha mencionado que existen tres tipos (tipo1 asociadas a anticuerpos antimembrana basal glomerular con o sin manifestaciones pulmonares, tipo 2 asociada a complejos inmunes y tipo 3 pauciinmunes con lesiones necrotizantes glomerulares), siendo las vasculitis asociadas a ANCA la principal etiología. Siempre que se presente este síndrome no podrán faltar los datos serológicos de anticuerpos anti-MBG, complejos inmunes circulantes, anticuerpos antinucleares ANA, anti-DNA, complemento, crioglobulinas y ANCA $(10,11)$.

\section{Buscar nexos por historia clínica}

Antecedentes familiares: una historia familiar de enfermedad renal puede orientar hacia causas congénitas de debut habitual en la infancia como síndrome de Alport, especialmente si se asocian a pérdida auditiva. También existen formas 
familiares de nefropatía por IgA, glomeruloesclerosis focal y segmentaria y síndrome hemolítico urémico (5).

\section{Historia farmacológica detallada}

Tabla 3 (16) (18).

Datos epidemiológicos y antecedente de infecciones.

Tabla 4 (19-34).

\section{Asociación con glomerulopatías paraneoplásicas}

Corresponden a manifestaciones raras de las enfermedades neoplásicas que no resultan fácilmente distinguibles del daño renal iatrogénico o de proteinuria por sobreflujo o sobreproducción (15). Las asociaciones con mayor relevancia epidemiológica son la nefropatía membranosa con tumores sólidos; enfermedad de cambios mínimos con malignidad hematológica, manifestándose en ambos casos
Tabla 3. Medicamentos asociados a enfermedad glomerular.

\begin{tabular}{|l|l|}
\hline Glomerulopatía & Medicamentos implicados \\
\hline Nefropatía membranosa & Penicilamina, sales de oro, IECAs, AINEs, mercurio \\
\hline Cambios mínimos & $\begin{array}{l}\text { AINEs, interferón a, y } \\
\text { Antibióticos: ampicilina, rifampicina } \\
\text { Metimazol, tamoxifen } \\
\text { vacunas }\end{array}$ \\
\hline Membranoproliferativa & $\begin{array}{l}\text { Ciclosporina, tacrolimus, mitomicina C, } \\
\text { anticonceptivos orales }\end{array}$ \\
\hline
\end{tabular}

con síndrome nefrótico con características atípicas, siendo la edad tardía de manifestación de enfermedad glomerular el principal signo de alarma. Otra consideración de riesgo de neoplasia asociada deberá hacerse particularmente en los casos de glomerulonefritis rápidamente progresiva por vasculitis asociadas a ANCA $(35,36)$. (Tabla 5)

Tabla 4. Asociación de hepatitis infecciosa y enfermedad glomerular

\begin{tabular}{|c|c|c|c|c|}
\hline Virus & Mecanismo & Complemento & Patrón histológico & Síndrome de presentación \\
\hline \multirow[t]{4}{*}{ VHB } & \multirow[t]{2}{*}{ Crioglobulinemia } & \multirow[t]{2}{*}{ Bajo } & Nefropatía membranosa & S. nefrótico \\
\hline & & & Nefropatía membranoproliferativa & S. nefrítico \\
\hline & \multirow[t]{2}{*}{ Poliangitis microscópica } & \multirow[t]{2}{*}{ Normal } & Glomerulonefritis crescéntica & Glomerulonefritis rápidamente progresiva \\
\hline & & & N. membranoproliferativa & S. nefrítico \\
\hline \multirow[t]{3}{*}{ VHC } & Crioglobulinemia & \multirow[t]{3}{*}{ Bajo } & Membranoproliferativa & S. nefrítico \\
\hline & Depósito complejos & & Membranosa & \multirow[t]{2}{*}{ S. nefrótico } \\
\hline & $\mathrm{Ag}-\mathrm{Ac}$ & & GEFS & \\
\hline \multirow[t]{3}{*}{ VIH } & Lesión directa & Normal & Glomeruloesclerosis focal y segmentaria colapsante & S. nefrótico \\
\hline & \multirow[t]{2}{*}{ Complejos inmunes } & \multirow[t]{2}{*}{ Bajo } & Membranoproliferativa & \multirow[t]{2}{*}{ Síndrome nefrítico/ PTT-SHU } \\
\hline & & & Nefropatía IgA secundaria & \\
\hline
\end{tabular}

Tabla 5. Glomerulopatía paraneoplásica.

\begin{tabular}{|c|c|c|c|}
\hline Glomerulopatía & Tumores asociados & Forma de presentación & Factores de riesgo/signos de alarma \\
\hline Nefropatía membranosa & $\begin{array}{l}\text { C. broncogénico } \\
\text { C. gástrico } \\
\text { C. renal } \\
\text { C. seno } \\
\text { C. próstata } \\
\text { C. colorrectal }\end{array}$ & Síndrome nefrótico & $\begin{array}{l}\text { Edad }>60 \text { años } \\
\text { Hombre } \\
\text { Fumador } \\
\text { Biopsia: }>8 \text { células } \\
\text { inflamatorias/glomérulo }\end{array}$ \\
\hline Enfermedad cambios mínimos & $\begin{array}{l}\text { L. Hodgkin } \\
\text { Mieloma múltiple } \\
\text { Timoma }\end{array}$ & Síndrome nefrótico & $\begin{array}{l}\text { Edad tardía presentación (la forma primaria } \\
\text { debuta antes de los } 20 \text { años) }\end{array}$ \\
\hline Nefropatía IgA secundaria/ PHS secundaria & $\begin{array}{l}\text { Carcinoma renal células claras } \\
\text { C. pulmón célula pequeña y no pequeña }\end{array}$ & Síndrome nefrítico & $\begin{array}{l}\text { Hombre } \\
\text { Edad }>60 \text { años } \\
\text { Poliartritis } \\
\text { Lesiones necrotizantes piel }\end{array}$ \\
\hline GEFS & Carcinoma renal células claras & Síndrome nefrótico & Edad $>50$ años \\
\hline
\end{tabular}




\section{3. ¿Qué datos de laboratorio y de imágenes pueden resultar útiles?}

Al abordar por síndromes la enfermedad glomerular y conocer las etiologías más frecuentemente relacionadas con cada síndrome, resulta evidente que no puede existir un listado único de ayudas diagnósticas a solicitar, sino que los exámenes solicitados, incluidos los perfiles inmunológico e infeccioso, deberán ser dirigidos por la sospecha clínica particular en cada paciente. Resultará útil la medición del complemento sérico, que más que una clasificación estática en glomerulonefritis hipocomplementémicas versus normocomplementémicas; ejemplo de ello se puede mencionar la presencia de síndrome nefrótico masivo sin hipertensión arterial ni datos de daño renal crónico que más probablemente sugiera una nefropatía membranosa; en la etiología primaria o idiopática de esta nefropatía están implicados anticuerpos que se dirigen contra un antígeno glomerular intrínseco específico del podocito, a diferencia los casos de etiología secundaria mediados por el depósito de inmunocomplejos en la membrana basal glomerular; características que explican que en ambos casos se observe un engrosamiento característico de la membrana basal glomerular, pero que los casos idiopáticos cursen con valores de complemento normal, a diferencia de las etiologías secundarias que cursaran con complemento consumido $(2,3,37)$ (Tabla 6).

\section{4. ¿Existe indicación de biopsia renal?}

Cuatro situaciones se consideran mandatarias de biopsia renal: 1. Síndrome nefrótico (excepto en niños por la alta frecuencia en ellos de enfermedad de cambios mínimos con curso clínico benigno y respuesta favorable a tratamiento con corticosteroides en monoterapia y excepto en presunción de nefropatía diabética); 2. Enfermedad renal en el contexto de enfermedad sistémica (lupus eritematoso sistémico, mieloma múltiple, amiloidosis, vasculitis); 3. Falla renal aguda en el contexto de enfermedad glomerular y glomerulonefritis rápidamente progresiva; 4. Anormalidades en sedimento urinario en receptor de transplante renal (rechazo de transplante versus enfermedad glomerular de novo o recurrente) La biopsia renal brinda información útil

Tabla 6. Clasificación de las lesiones glomerulares en función del complemento.

\begin{tabular}{|c|c|c|}
\hline Etiología & Complemento disminuido & Complemento normal \\
\hline Primaria & $\begin{array}{l}\text { Membranoproliferativa I (50\%) } \\
\text { Membranoproliferativa II }(80 \%)\end{array}$ & $\begin{array}{l}\text { GEFS } \\
\text { N. membranosa } \\
\text { Enfermedad cambios mínimos } \\
\text { Nefropatía IgA } \\
\text { Rápidamente progresiva idiopática }\end{array}$ \\
\hline Secundaria & $\begin{array}{l}\text { Posestreptocócica (90\%) } \\
\text { Endocarditis subaguda (90\%) } \\
\text { LES (focal 75\%, difusa 90\%) } \\
\text { Crioglobulinemia (85\%) }\end{array}$ & $\begin{array}{l}\text { Poliangitis microscópica } \\
\text { Granulomatosis de Wegener } \\
\text { Vasculitis hipersensibilidad } \\
\text { Púrpura Henoch- Schonlein } \\
\text { Síndrome Goodpasture }\end{array}$ \\
\hline
\end{tabular}

para el diagnóstico etiológico y para el pronóstico y, debe emplearse siempre que su resultado derive en poder definir diversas conductas terapéuticas. Siempre que se realice este procedimiento, el reporte debe incluir los hallazgos a la microscopía de luz (describe la celularidad glomerular y clasifica el compromiso en focal o difuso), microscopía electrónica (describe la presencia y localización subcelular de complejos inmunes, grado de daño celular, consistencia de la membrana basal y cambios en la ultraestructura del podocito) e inmunofluorescencia (determina la presencia o ausencia de un proceso inmune de base con coloraciones dirigidas contra anticuerpos específicos y componentes del complemento, además de determinar presencia de proteínas de matriz, material amiloide o inclusiones virales).

\section{Conclusiones}

El reconocimiento temprano de la enfermedad glomerular es de gran importancia en prevenir daño renal irreversible. La presentación clínica brinda información valiosa para identificar la causa y poder definir un tratamiento adecuado y oportuno, además de brindar información útil para hacer un abordaje diagnóstico lógico y práctico que repercutirá no sólo en el paciente sino en los costos de atención en salud.

\section{Referencias}

1. Chadban S.J. and R.C. Atkins, Glomerulonephritis. Lancet 2005; 365(9473): 1797-806.

2. Segelmark M. and T. Hellmark. Autoimmune kidney diseases. Autoimmun Rev 2010; 9(5): 366-71.

3. Madaio M.P. and J.T. Harrington. The diagnosis of glomerular diseases: acute glomerulonephritis and the nephrotic syndrome. Arch Intern Med 2001; 161(1): 25-34.

4. Hull R.P. and D.J. Goldsmith. Nephrotic syndrome in adults. BMJ 2008; 336(7654): 1185-9.

5. Wiggins R.C. The spectrum of podocytopathies: a unifying view of glomerula diseases. Kidney Int 2007. 71(12): 1205-14.

6. Molino C., F. Fabbian, and C. Longhini. Clinical approach to lupus nephritis: recent advances. Eur J Intern Med 2009. 20(5): 447-53.

7. Hricik D.E., M. Chung-Park, and J.R. Sedor, Glomerulonephritis. $N$ Eng $J$ Med, 1998. 339(13): 888-899.

8. Khanna R. Clinical presentation \& management of glomerular diseases: hematuria, nephritic \& nephrotic syndrome. Mo Med 108(1): 33-6.

9. Beck L.H., Jr. and D.J. Salant. Glomerular and tubulointerstitial diseases. Prim Care 2008. 35(2): 265-96.

10. Couser W.G. Rapidly progressive glomerulonephritis: classification, pathogenetic mechanisms, and therapy. Am J Kidney Dis 1988; 11(6): 449-64.

11. Angangco R., et al. Does truly 'idiopathic' crescentic glomerulonephritis exist? Nephrol Dial Transplant 1994; 9(6): 630-6.

12. Grossfeld G.D., et al. Asymptomatic microscopic hematuria in adults: summary of the AUA best practice policy recommendations. Am Fam Physician 2001; 63(6): 1145-54.

13. Chun M.J., et al. Focal segmental glomerulosclerosis in nephrotic adults: presentation, prognosis, and response to therapy of the histologic variants. J Am Soc Nephrol 2004; 15(8): 2169-77.

14. Cameron J.S. Focal segmental glomerulosclerosis in adults. Nephrol Dial Transplant 2003; 18 (Suppl 6): vi45-51.

15. Bacchetta J., et al. Paraneoplastic glomerular diseases and malignancies. Crit Rev Oncol Hematol 2009; 70(1): 39-58.

16. Sethi S. and F.C. Fervenza. Membranoproliferative glomerulonephritis--a new look at an old entity. N Engl J Med 2012; 366(12): 1119-31.

17. Donadio J.V. and J.P. Grande. IgA nephropathy. N Engl J Med 2002; 347(10): 738-48.

18. Glassock R.J. Secondary minimal change disease. Nephrol Dial Transplant 2003; 18 (Suppl 6): vi52-8 
19. Lai F.M., et al. Hepatitis B virus-related nephropathy and lupus nephritis: morphologic similarities of two clinical entities. Mod Pathol 2000; 13(2): 166-72.

20. Lai K.N., et al. Membranous nephropathy related to hepatitis B virus in adults. N Engl J Med 1991; 324(21): 1457-63.

21. Chan T.M. Hepatitis B and Renal Disease. Curr Hepat Rep 2010; 9(2): 99-105.

22. Kamar N., et al. Hepatitis C virus-related kidney disease: an overview. Clin Nephrol 2008; 69(3): 149-60.

23. Khattab M.A., M. Eslam, and S.M.Alavian. Hepatitis $C$ virus as a multifaceted disease: a simple and updated approach for extrahepatic manifestations of hepatitis C virus infection. Hepat Mon 2010; 10(4): 258-69.

24. Perico N., et al. Hepatitis C infection and chronic renal diseases. Clin J Am Soc Nephrol 2009; 4(1): 207-20.

25. Pipili C., G. Ilonidis, and E. Cholongitas. Hepatitis C virus and kidney: a strong association with different clinical aspects. Liver Int 2011; 31(8): 1071-80.

26. Saddadi F., et al. Renal involvement in patients with hepatitis $\mathrm{C}$ virus infection. Iran J Kidney Dis 2010; 4(2): 123-7.

27. Zignego A.L., et al. The hepatitis $\mathrm{C}$ virus infection as a systemic disease. Intern Emerg Med 2012; 7(Suppl 3): 201-8.

28. Herman, E.S. and P.E. Klotman. HIV-associated nephropathy: Epidemiology, pathogenesis, and treatment. Semin Nephrol 2003; 23(2): 200-8.
29. Kaufman L., S.E. Collins, and P.E. Klotman, The pathogenesis of HIV-associated nephropathy. Adv Chronic Kidney Dis 2010; 17(1): 36-43.

30. Medapalli R.K., J.C. He, and P.E. Klotman. HIV-associated nephropathy: pathogenesis. Curr Opin Nephrol Hypertens 2011; 20(3): 306-11.

31. Wyatt C.M., P.E. Klotman, and V.D. D'Agati. HIV-associated nephropathy: clinical presentation, pathology, and epidemiology in the era of antiretroviral therapy. Semin Nephrol 2008; 28(6): 513-22.

32. Wyatt C.M., K. Meliambro, and P.E. Klotman. Recent progress in HIVassociated nephropathy. Annu Rev Med 2012; 63: 147-59.

33. Wyatt C.M, et al. The spectrum of kidney disease in patients with AIDS in the era of antiretroviral therapy. Kidney Int 2009; 75(4): 428-34.

34. Wyatt C.M, P.E. Rosenstiel, and P.E. Klotman. HIV-associated nephropathy. Contrib Nephrol 2008; 159: 151-61.

35. Lefaucheur C., et al. Membranous nephropathy and cancer: Epidemiologic evidence and determinants of high-risk cancer association. Kidney Int 2006; 70(8): 1510-7.

36. Lien Y.H. and L.W. Lai. Pathogenesis, diagnosis and management of paraneoplastic glomerulonephritis. Nat Rev Nephrol 2011; 7(2): 85-95.

37. Hebert L.A., F.G. Cosio, and J.C. Neff. Diagnostic significance of hypocomplementemia. Kidney Int 1991; 39(5): 811-21. 